\title{
Analysis of risk factors on prognosis of Acinetobacterbaumannii bloodstream infection
}

\author{
Qiao L, Zhang JS*, Mei YN, Zhang HZ and Su CL \\ Department of Emergency Center, First Affiliated Hospital of Nanjing Medical University, Nanjing 210029, Jiangsu, China
}

\begin{abstract}
Objective: To investigate the predictors associated with mortality for patients with Acinetobacterbaumannii bloodstream infections.

Methods: A retrospective study was performed on 115 adult patients with A. baumannii bloodstream infections, who were admitted to Chinese PLA General Hospital from January 2009 to December 2011. And the risk factors, such as age, gender, mechanical ventilation, concomitant conditions, invasive procedures, use of antibiotics prior to isolation and antimicrobial susceptibility, were analyzed.

Results: Among 155 patients, 45 died, with mortality of 39.1\%. Mechanical ventilation (OR=8.8, 95\%CI 2.6-29.7, $\mathrm{P}=0.000)$, prior immunosuppression $(\mathrm{OR}=9.4$, $95 \% \mathrm{CI} 2.2-40.7, \mathrm{P}=0.003)$, use of carbapenems prior to isolation ( $\mathrm{OR}=4.5$, 95\% CI 1.4-14.2, $\mathrm{P}=0.011$ ) were the risk factors associated with mortality for patients with A. baumannii bloodstream infections.

Conclusion: Independent risk factors which caused the death of patients suffered from Acinetobacterbaumannii bloodstream infections are mechanical ventilation, prior immunosuppression and use of carbapenems prior to isolation, while multi-drug resistance of strains is not the risk factor.
\end{abstract}

\section{Introduction}

Bloodstream infection is one of the most serious performance of infectious diseases, with rapid development and high mortality. As the interventional diagnosis and treatment therapy has been widely utilized and various broad-spectrum drugs been used, incidence of acquired bloodstream infection in hospital increased year by year. Widely spread among hospitals, Acinetobacterbaumannii is a major pathogenic bacteria causing acquired infection. It can be resistant to a variety of antibacterial drugs in a very short time, which poses great threat to clinical anti-infective treatment. In recent years, detection rate of A. baumannii has shown an upward tendency. High mortality of bloodstream infection caused by such bacteria has attracted much attention clinically. Therefore, understanding risk factors on prognosis of A. baumannii infection patients is significant to identify high-risk patients promptly, enhance clinical treatment and improve prognosis of patients. This paper conducted a retrospective analysis on patients with A. baumannii from January 2009 to December 2011 and investigated risk factors on prognosis of A. baumannii infection patients. Reported as follows.

\section{Materials and methods}

\section{Materials}

A. baumannii patients who were admitted to Chinese PLA General Hospital from Jan 2009 to Dec 2011 were objects of study. Selected cases and samples conformed to Diagnostic Criteria of Nosocomial Infection published by Ministry of Health in 2001. Repetitive strains from the same patient with the same disease were eliminated. These objects were divided into death group and survival group based on disease outcome of patients. Among them, there were 45 cases in death group, 33 males and 12 females with average age at $(46.5 \pm 20.2)$; 70 cases in survival group, 47 males and 23 females, with average age at (55.0 \pm 18.7$)$.
Isolation, culture and appraisal of strains and antimicrobial susceptibility test

All blood samples were isolated, cultured and appraised according to hospital routine methods. Vitro antimicrobial susceptibility test was performed by using K-B method recommended by CLSI, which in corresponding years was set as criteria for results evaluation and interpretation. Intermediate strains, according to antimicrobial susceptibility test, were drug resistance. Escherichia coli (E. coil) ATCC25922 and standard strains of pseudomonas aeruginosa ATCC27853 were utilized for quality control.

\section{Methods}

Performed retrospective study and collected patients' clinical materials, including general information, basic diseases, invasive procedures, use of antibiotics prior to isolation antimicrobial susceptibility test results as well as clinical disease outcome.

\section{Statistical analysis}

SPSS 13.0 software was employed for statistical description and analysis. Quantitative date were measured by $\mathrm{x} \pm \mathrm{s}$; group comparison was carried out by using T test, T' test or rank-sum test. Qualitative materials were expressed as percentage; group comparison was detected by $\chi^{2}$ test or Fisher's extract probability method. Variables with significant difference in univariate analysis would be measured by

Correspondence to: Zhang JS, Department of Emergency Center, First Affiliated Hospital of Nanjing Medical University, Nanjing 210029, Jiangsu, China; Tel: (852)2609-8054; Fax: (852)2609-8054; E-mail:zhangjso@sina.com

Key words: Acinetobacterbaumannii; bloodstream infection; risk factors on prognosis; multi-drug resistance

Received: July 30, 2016; Accepted: August 26, 2016; Published: August 29, 2016 
Logistic regression analysis, $\mathrm{p}<0.05$ with statistical meaning

\section{Results}

\section{Materials}

In total of $115 \mathrm{~A}$. baumannii bloodstream infection strains were detected. 115 infected patients ranged in age from 17 to 94 , with average age at $51.7 \pm 19.8$. Among them, there were 80 males (69.6\%), 35 females (30.4\%); 50 ICU patients (43.5\%), 65 non-ICU patients (56.5\%); 97 patients with invasive procedure $(84.3 \%) ; 83$ patients with previous surgeries $(72.2 \%) ; 60$ patients with mechanical ventilation $(52.2 \%)$. 103 patients $(89.6 \%)$ received treatment of use of antibiotics prior to isolation. Among them, 66 patients with antibiotics of carbapenems (57.4\%); 63 patients with antifungal agents (54.8\%); 54 patients with ß-lactam antibiotics/ ß-lactamase inhibitor mixture antibiotics (47.0\%); types of antibiotics $0-9$, average at $3.7 \pm 2.2,79$ patients with combined use of 3 or more antimicrobial drugs (68.7\%). The number of A. baumannii with feature of multi-drug resistances was 95 (82.6\%). Among all 155 patients with A. baumannii bloodstream infection, 45 patients died (39.1\%). See Table 1.

\section{Analysis on drug resistance of A. baumannii against antimicrobial drugs}

Drug resistance rate of A. baumannii strains from blood of 115 patients was relatively high. In 2009, 2010 and 2011, sensitivity rate of all antibacterial drugs, including carbapenems, was no more than $35 \%$. Meropenem had the highest sensitivity rate (23.5\%), and its change of such rate in those three years was $15.9 \%$ (7/44), 30.2\% (13/43), and $25.0 \%(7 / 28)$ respectively. See Table 2. Compared with strains in survival group, drug resistance rates of strains in death group against amikacin, ceftazidime, ciprofloxacin and SMZ-TMP were relatively

Table 1. Characteristics of 115 Patients with Acinetobacter baumannii Bloodstream Infections.

\begin{tabular}{|l|c|}
\hline Characteristics & Value \\
\hline Mean age (years) & $51.7(17-94)$ \\
Male gender & $80(69.6 \%)$ \\
Intensive-care unit admission & $50(43.5 \%)$ \\
Year of isolation & \\
2009 & $44(38.3 \%)$ \\
2010 & $43(37.4 \%)$ \\
2011 & $28(24.3 \%)$ \\
Any concomitant conditions & \\
Mechanical ventilation & $60(52.2 \%)$ \\
Invasive procedures & $97(84.3 \%)$ \\
prior immunosuppression & $21(18.3 \%)$ \\
Previous surgeries & $83(72.2 \%)$ \\
Trauma & $17(14.8 \%)$ \\
organ failure at onset of bacteremia & $30(26.1 \%)$ \\
Use of antibiotics prior to isolation & \\
any & $103(89.6 \%)$ \\
third-generation cephalosporins & $16(13.9 \%)$ \\
B-lactam/B-lactamase inhibitor & $54(47.0 \%)$ \\
Carbapenems & $66(57.4 \%)$ \\
Aminoglycosides & $17(14.8 \%)$ \\
Quinolones & $24(20.9 \%)$ \\
Antifungal agent & $63(54.8 \%)$ \\
Other antibiotics & $89(77.4 \%)$ \\
length of B-lactam/B-lactamase inhibitor use $\geq 3$ days & $40(34.8 \%)$ \\
length of third-generation cephalosporins use $\geq 3$ days & $7(6.1 \%)$ \\
length of Carbapenems use $\geq 3$ days & $59(51.3 \%)$ \\
length of Quinolones use $\geq 3$ days & $16(13.9 \%)$ \\
length of Antifungal agent use $\geq 3$ days & $58(50.4 \%)$ \\
Days of prior antibiotic therapy & $25.0(0-70)$ \\
No. of prior antibiotic groups used & $3.7(0-9)$ \\
No. of prior antibiotic groups used $\geq 3$ & $79(68.7 \%)$ \\
Multidrug-resistant strains & $95(82.6 \%)$ \\
Mortality & $45(39.1 \%)$ \\
\hline & \\
\hline
\end{tabular}

Table 2. In vitro antimicrobial susceptibility analysis of Acinetobacter baumannii isolates.

\begin{tabular}{|l|l|l|l|l|}
\hline Antibiotic agent & Overall & $\mathbf{2 0 0 9}$ & $\mathbf{2 0 1 0}$ & $\mathbf{2 0 1 1}$ \\
\hline Amikacin & 19.1 & $9.1(4 / 44)$ & $30.2(13 / 43)$ & $17.9(5 / 28)$ \\
\hline Cefotaxime & 1.7 & $2.3(1 / 44)$ & $2.3(1 / 43)$ & - \\
Ceftazidime & 17.4 & $9.1(4 / 44)$ & $20.9(9 / 43)$ & $25.0(7 / 28)$ \\
Cefuroxime & 0.9 & - & $2.3(1 / 43)$ & - \\
Ciprofloxacin & 16.5 & $9.1(4 / 44)$ & $18.6(8 / 43)$ & $25.0(7 / 28)$ \\
Meropenem & 23.5 & $15.9(7 / 44)$ & $30.2(13 / 43)$ & $25.0(7 / 28)$ \\
Piperacillin/tazobactam & 9.6 & $4.5(2 / 44)$ & $9.3(4 / 43)$ & $17.9(5 / 28)$ \\
$\begin{array}{l}\text { Sufamethoxazole/ } \\
\text { trimethoprim }\end{array}$ & 15.7 & $9.1(4 / 44)$ & $16.3(7 / 43)$ & $25.0(7 / 28)$ \\
\hline
\end{tabular}

Table 3. Antimicrobial susceptibility rates of A.baumannii isolates.

\begin{tabular}{|l|l|l|l|l|}
\hline Antibiotic & Total[n(\%)] & Survivors[n(\%)] & Nonsurvivors[n(\%)] & P \\
\hline Amikacin & $22(19.1)$ & $19(27.1)$ & $3(6.7)$ & 0.006 \\
\hline Cefotaxime & $2(1.7)$ & $2(2.9)$ & - & - \\
Ceftazidime & $20(17.4)$ & $17(24.2)$ & $3(6.7)$ & 0.015 \\
Cefuroxime & $1(0.9)$ & $1(1.4)$ & - & - \\
Ciprofloxacin & $19(16.5)$ & $16(22.9)$ & $3(6.7)$ & 0.023 \\
Meropenem & $27(23.5)$ & $19(27.1)$ & $8(17.8)$ & 0.248 \\
Piperacillin/tazobactam & $11(9.6)$ & $9(12.9)$ & $2(4.4)$ & 0.241 \\
$\begin{array}{l}\text { Sufamethoxazole/ } \\
\text { trimethoprim }\end{array}$ & $18(15.7)$ & $16(22.9)$ & $2(4.4)$ & 0.008 \\
\hline
\end{tabular}

high, difference with statistical meaning $(\mathrm{p}<0.05)$. See Table 3.

Univariate analysis on risk factors on prognosis of $\mathrm{A}$. baumannii bloodstream infection

Average ages of patients in death group and survival group were $46.5 \pm 20.2$ and $55.0 \pm 18.7$ respectively, difference with statistical meaning $(p<0.05)$. Patients in death group were mostly checked in ICU wards $\left(\chi^{2}=8.212, P<0.05\right)$. Except for invasive procedure and trauma, factors such as reception of mechanical ventilation, prior immunosuppression, previous history of surgery and organ failure were different in expression in two groups $(\mathrm{p}<0.05)$. Compared with survival group, death group was longer in time for receiving use of antibiotics prior to isolation and more in variety of antibacterial drugs. Empirical treatment of carbapenems, quinolones and antifungal agents had big difference in two groups $(\mathrm{p}<0.05)$. In death group, percentage of multi-drug resistant strains was higher $(\mathrm{p}<0.05)$.

\section{Logistic regression analysis on risk factors}

Performed multi-variate non conditional regression analysis on factors of statistical significance from the result of univariate analysis. The result shown that mechanical ventilation, prior immunosuppression, previous history of surgery and use of carbapenems prior to isolation are risk factors of patients of A. baumannii bloodstream infection.

\section{Discussion}

A. baumannii is non-fermentative Gram-negative bacilli (NFGNB). It is strong in viability and has no special requirement for nutrition $[1,2]$. It is widely distributed in human skin, respiratory passage, digestive tract, urogenital tract and hospital, and can survive for a long time [3]. Its colonization rate in patients can reach $75 \%$ [4]. In 1970s, as pathogen that could infect patients, it was rarely to be seen. However, in recent ten years, reports on infection caused by A. baumannii has increased, for example, acquired pneumonia, bacteremia, urinary infection and wound infection etc; while that patients of bloodstream infection are many in number, serious in condition and high in mortality has drawn clinical focus $[5,6]$.

In previous studies, risk factors of A. baumannii bloodstream infection were as follows: serious underlying illnesses, exposure of 
Table 4. Univariate analysis of predictors associated with mortality for patients with Acinetobacter baumannii bloodstream infections.

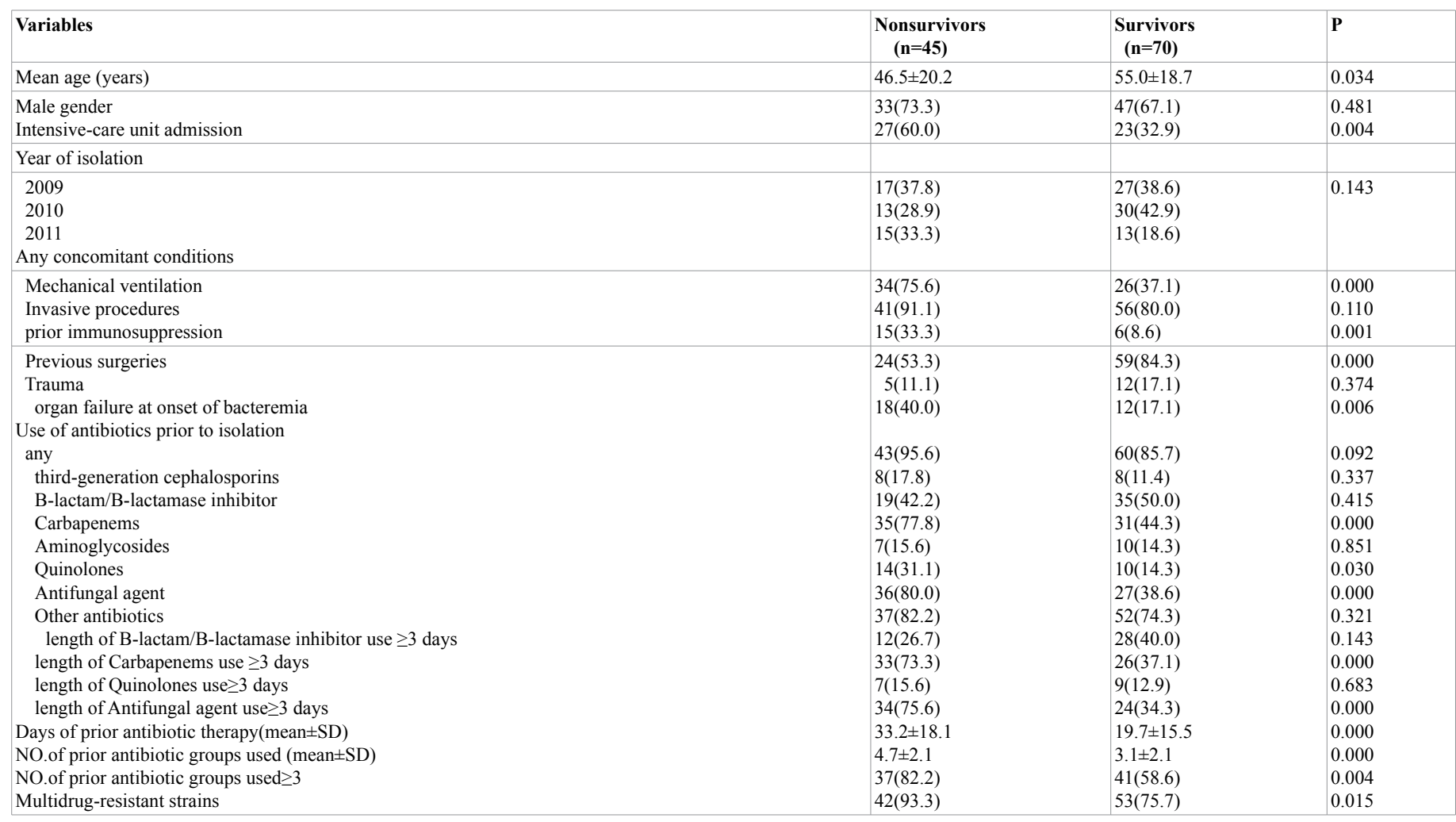

Table 5. Multivariate analysis of predictors associated with mortality for patients with Acinetobacter baumannii bloodstream infections.

\begin{tabular}{|l|c|c|c|}
\hline Variables & P & OR & $\mathbf{9 5 \% C I}$ \\
\hline Mechanical ventilation & 0.000 & 8.758 & $2.582-29.702$ \\
\hline Surgery & 0.002 & 0.132 & $0.038-0.462$ \\
\hline prior immunosuppression & 0.003 & 9.410 & $2.177-40.679$ \\
use of carbapenems prior to isolation & 0.011 & 4.473 & $1.409-14.194$ \\
constant & 0.014 & 0.196 & \\
\hline
\end{tabular}

OR, odds ratio; $\mathrm{CI}$, confidence interval.

antibacterial drugs, colonization of bacteria, history of surgery, central venous catheter and indwelling catheter, parenteral alimentation, mechanical ventilation as well as time in ICU [7]. Hilmar Wisplinghoff et al performed retrospective study on 111 patients of A. baumannii bloodstream infection and 2952 patients of bloodstream infection caused by other Gram-negative bacilli and found that case group and control group were different in factors including history of trauma, ratio of time in ICU, mechanical ventilation, artery catheter and indwelling catheter and such difference had statistical meaning [8]. In this study, among 115 patients of A. baumannii bloodstream infection, 50 patients (43.5\%) were checked in ICU; 97 (84.3\%) received various invasive procedure; 60 (52.2\%) received mechanical ventilation; 83 (72.2\%) with previous history of surgery; 103 (89.6\%) received treatment of empirical antibiotics before diagnosed with bloodstream infection; 79 (68.7\%) took 3 or more antibiotics. Percentage of patients who used antibiotics of carbapenems class was the highest, with 66 (57.4\%).

Under the selective pressure of antibiotics, A. baumannii can constantly acquire a variety of drug resistance mechanism, which leads to serious problem of drug resistance, multi-drug resistance and even pan-drug resistance. Statistics from CHINET in 2010 stated that resistance rates of Acinetobacter sp. bacteria to cefoperazone sulbactam and minocycline were $29.9 \%$ and $33.3 \%$ respectively, nearly half or above to other tested drugs, $75 \%$ to carbapenems antibiotics [9]. Drug resistance of A. baummannii in this research was relatively high, with more than $65 \%$ to 8 antimicrobial drugs. Among them, sensitivity rate of meropenem in 2009, 2010, and 2011 were $15.9 \%, 30.2 \%$ and $25.0 \%$ respectively. But data shown that resistance rates of isolated strains against meropenem were similar in death and survival group.

Multi-variate analysis in this research indicated that mechanical ventilation, prior immunosuppression, previous history of surgery and use of carbapenems prior to isolation are risk factors of patients of A. baumannii bloodstream infection, which is not completely consistent with conclusion of Qiao Li. This is probably related to study area and population [10]. Patients who require mechanical ventilation are serious condition. But mechanical ventilation would destroy normal defense barrier of host, provide conditions for colonization of bacteria, and even superinduce ventilator associated pneumonia and thus seriously affect prognosis. In literature, mortality of patients of severe VAP infection can be $50 \%$ to $70 \%$ [11]. It was pointed out in related reports that patients with prior immunosuppression are easy to suffer from bloodstream infection and their prognosis are usually critical [12]. Chiang D H and L. Silvia Munoz et al also found that tumor underlying disease is risk factor causing death of patients with A. baumannii bloodstream infection [13,14]. Data in this research revealed that use of carbapenems prior to isolation would increase risk of death of patients. Cunha et al considered that imipenem has high drug resistance potential. Carbapenems antibiotics can not only make A. baumannii resistant to carbapenems but also increase resistance rate of it against aminoglycoside, fluoroquinolones (FQNs) and B-lactam antibiotics [15]. Such characteristic of carbapenem antibiotics might be correlated to its increasing death risk of patients, but it is not clear so far and further studies to be done. Besides, this study found previous history of surgery is protective factor of patients prognosis of A. 
baumannii bloodstream infection (OR 0.132, 95\% CI 0.038-0.462), but its reason is yet to be explained.

It is usually considered that mortality of multi-drug resistant A. baumannii bloodstream infection is relatively high $[16,17]$. But in this paper, univariate analysis indicated that difference of multi-drug resistance in death and survival group had statistical significance. But in multi-variate analysis, multi-drug resistance is not risk factor causing death of patients of A. baumannii bloodstream infection. Qiao $\mathrm{Li}$ in China also found drug resistance of bacteria has no evident impact on prognosis. She thought that because of limit to distinguishing traditional micro-organism in laboratory, A. baumannii, acinetobacter genotype 3 and acinetobacter gene $13 \mathrm{TU}$ cannot be differentiated clinically. But their difference in drug resistance mechanism, drug tolerance and prognosis would perhaps affect accuracy of research result [10]. However, after studying risk factors on prognosis of bloodstream infection patients caused by above mentioned three gene of acinetobacter, Yi-Chieh Lee found that A. baumannii, multi-drug resistance and appropriate treatment of antibiotics were associated, in univariate analysis, with 30-days mortality. But multi-variate analysis proved that they were not risk factors of 30-days mortatlity. Instead, underlying disease and severity of bacteremia affected prognosis [18]. Seung-Kwan Lim also agreed that host factors, for example, concomitant disease, multiple organ failure and prior immunosuppression, had greater effects on prognosis of A. baumannii bloodstream infection patients. In his research, high score of APACHE II was the only significant risk factor of prognosis death of patients [19]. Some scholars studied risk factors of imipenem A. baumannii bloodstream infection and discovered that mortality was quite different between drug resistance group and drug sensitivity group. But after adjusting mixed factors like severity of underlying diseases, inappropriate antibiotics treatment and bloodstream infection sources in multi-variate analysis, difference of mortality in these two groups had not statistical significance [20]. On one hand, patients in severe condition are easy to be infected by multi-drug resistance A. baumannii, and their mortality was at high degree originally. Blot et al reported that mortality of A. baumannii bloodstream infection was only $7.8 \%$; among severe patients, such infection had nothing to do with mortality ${ }^{[21]}$. On the other, although A. baumannii is not sensitive to a majority of antibiotics, its virulence is not high. Some scholars pointed out that virulence of drug resistant bacteria does not increase, so mortality might not enhance. Underlying diseases of patients and severity of disease this time ultimately affect prognosis $[11,22,23]$. But to have assured conclusion, more perspective studies should be rigorously designed.

However, this paper has its limitation: 1. single center research. Besides, prevalence of A. baumannii is different in region, so results of this study might not be applied to other hospitals; 2 . Retrospective research.Lack of quantitative index (for example, APACHE II score) for severity of diseases and prognosis. But this study collected and analyzed several related indexes, such as mechanical ventilation and prior immunosuppression etc.

Mechanical ventilation, prior immunosuppression and use of carbapenems prior to isolation are the risk factors on prognosis of Acinetobacterbaumannii bloodstream infections, while multi-drug resistance of strains is not major factor affecting patients on prognosis. Thus, clinicians should focus on identifying severe patients and treat adequately in time to reduce their death risk.

\section{References}

1. Bergogne-Berezin E, Towner KJ (1996) Acinetobacter spp. as nosocomial pathogens: microbiological, clinical, and epidemiological features. Clin Microbiol Rev 9: 148-65.
2. Corbella X, Montero A, Pujol M (2000) Emergence and rapid spread of carbapenem resistance during a large and sustained hospital outbreak of multiresistant Acinetobacter baumannii. J Clin Microbiol 38: 4086-95.

3. Xu CH, Wang Z, Wang J. (2012) Control of Acinetobacter baumannii infection of ICU patients. Chinese Journal of Disinfection.

4. Lei H. Study on imipenem Acinetobacter baumannii genotype OXA and molecular epidemiology. Chief editor: Huang LJ, Yuan J (2012) Military Preventative Medicine.

5. Chen SJ, Chao TF, Chiang MC (2011) Predictors of mortality in surgical patients with Acinetobacter baumannii bacteremia. J Microbiol Immunol Infect 44: 209-14.

6. Lee NY, Chang TC, Wu CJ (2010) Clinical manifestations, antimicrobial therapy, and prognostic factors of monomicrobial Acinetobacter baumannii complex bacteremia. $J$ Infect 61: 219-27.

7. Brahmi N, Beji O, Abidi N (2007) Epidemiology and risk factors for colonization and infection by Acinetobacter baumannii in an ICU in Tunisia, where this pathogen is endemic. J Infect Chemother 13: 400-4

8. Wisplinghoff H, Edmond MB, Pfaller MA, Jones RN, Wenzel RP, Seifert H (2000) Nosocomial bloodstream infections caused by Acinetobacter species in United States hospitals: clinical features, molecular epidemiology, and antimicrobial susceptibility. Clin Infect Dis 31: 690-7.

9. Li GH, Zhu DM, Wang F (2012) Distribution and drug resistance of pathogens of bloodstream infection by CHINET in 2010. Chinese Journal of Infection and Chemotherapy.

10. Qiao L, Zhang JS, Mei YN, Zhang HZ, Su CL (2013) [Analysis of risk factors on prognosis of Acinetobacter baumannii bloodstream infection]. Zhonghua wei zhong bing ji jiu yi xue 25: 471-4.

11. Qiao ZH (2011) Analysis of risk factors on prognosis of ICU patients of pan- imipenem Acinetobacter baumannii bloodstream infection. China Medical Herald.

12. Papagheorghe R (2012) Bloodstream infections in immunocompromised hosts. Roum Arch Microbiol Immunol 71: 87-94.

13. Chiang DH, Wang CC, Kuo HY (2008) Risk factors for mortality in patients with Acinetobacter baumannii bloodstream infection with genotypic species identification. J Microbiol Immunol Infect 41: 397-402.

14. Munoz-Price LS, Zembower T, Penugonda S (2010) Clinical outcomes of carbapenemresistant Acinetobacter baumannii bloodstream infections: study of a 2-state monoclonal outbreak. Infect Control Hosp Epidemiol 31: 1057-1062.

15. Cunha BA (2001) Effective antibiotic-resistance control strategies. Lancet 357: 1307-8

16. Wareham DW, Bean DC, Khanna P (2008) Bloodstream infection due to Acinetobacter spp: epidemiology, risk factors and impact of multi-drug resistance. Eur $J$ Clin Microbiol Infect Dis 27: 607-612.

17. Kwon KT, Oh WS, Song JH (2007) Impact of imipenem resistance on mortality in patients with Acinetobacter bacteraemia. J Antimicrob Chemother 59: 525-530.

18. Lee YC, Huang YT, Tan CK (2011) Acinetobacter baumannii and Acinetobacter genospecies $13 \mathrm{TU}$ and 3 bacteraemia: comparison of clinical features, prognostic factors and outcomes. J Antimicrob Chemother 66: 1839-1846.

19. Lim SK, Lee SO, Choi SH (2011) The outcomes of using colistin for treating multidrug resistant Acinetobacter species bloodstream infections. J Korean Med Sci 26: 325-331.

20. Jamulitrat S, Arunpan P, Phainuphong P (2009) Attributable mortality of imipenemresistant nosocomial Acinetobacter baumannii bloodstream infection. J Med Assoc Thai 92: 413-419.

21. Blot S, Vandewoude K, Colardyn F (2003) Nosocomial bacteremia involving Acinetobacter baumannii in critically ill patients: a matched cohort study. Intensive Care Med 29: 471-475.

22. Levin AS, Levy CE, Manrique AE, Medeiros EA, Costa SF (2003) Severe nosocomial infections with imipenem-resistant Acinetobacter baumannii treated with ampicillin sulbactam. Int J Antimicrob Agents 21: 58-62.

23. Tomas M D M, Cartelle M, Pertega S (2005) Hospital outbreak caused by a carbapenemresistant strain of Acinetobacter baumannii : patient prognosis and risk-factors for colonisation and infection. Clinical Microbiology \& Infection the Official Publication of the European Society of Clinical Microbiology \& Infectious Diseases, 11: 540-546.

Copyright: (C2016 Qiao L. This is an open-access article distributed under the terms of the Creative Commons Attribution License, which permits unrestricted use, distribution, and reproduction in any medium, provided the original author and source are credited. 